\title{
PROCESS AND SYSTEMS Attitudes, experiences, and safety behaviours of adolescents and young adults who read visit notes: Opportunities to engage patients early in their care
}

\author{
Authors: Barbara D Lam, ${ }^{A}$ Fabienne Bourgeois, ${ }^{B}$ Catherine M DesRoches, ${ }^{C}$ Zhiyong Dong ${ }^{D}$ and Sigall K Bell ${ }^{C}$
}

\begin{abstract}
The Cures Act made access to electronic visit notes ('open notes') nearly universal across the USA, and efforts to share open notes with patients are underway worldwide. This landmark policy change provides an opportunity to engage adolescents and young adults (AYA) early in their care, yet little is known about their attitudes related to reading notes. We compared the responses of 332 AYA (13-25 years old) and 6,914 adults ( $>25$ years old) in a 2016 survey at two USA academic adult and paediatric hospitals. Over $85 \%$ of AYA and adults with available notes reported reading at least one note in the prior year. AYA reported similar benefits from note-reading to adults in 15 outcomes related to engagement, relational effects and safety behaviours, supporting efforts to engage AYA as partners in their care using open notes.
\end{abstract}

KEYWORDS: patient safety, open notes, adolescents and young adults

DOI: $10.7861 /$ fhj.2021-0118

\section{Introduction}

In the USA, the Office of the National Coordinator for Health Information Technology's Cures Act has mandated electronic access to clinical notes free of charge this past year, expanding 'open notes' to every patient in the country ${ }^{1}$ and marking a new era of digital health focused on transparency and accessibility. While this may seem a dramatic evolution for healthcare, the change may be welcomed or even expected by a younger generation of patients who have grown up immersed in technology. Inviting young patients to read their health information may give them an opportunity to step up as partners in their care. ${ }^{2-4}$ Studies of adult patients and parents of paediatric

Authors: ${ }^{\text {A }}$ clinical fellow, Beth Israel Deaconess Medical Center, Boston, MA, USA; ' assistant professor of pediatrics, Boston Children's Hospital, Boston, MA, USA; ' associate professor of Medicine, Beth Israel Deaconess Medical Center, Boston, MA, USA; ${ }^{\text {biostatistician, }}$ Beth Israel Deaconess Medical Center, Boston, MA, USA patients have shown that reading notes can improve safety behaviours and understanding of their care. ${ }^{5}$ Little is known, however, about how adolescents and young adults interact with their notes. ${ }^{6,7}$ Better understanding their perspectives can highlight opportunities to activate this patient population at a pivotal time, as they transition from paediatric to adult care, learn to make independent care decisions and begin to engage as safety partners with clinicians.

Patients who are 13-25 years of age, including adolescents and young adults (AYA), make up the majority of Generation Z and have some overlap with Millennials. Technology has been part of this generational cohort's lives since their childhoods and these 'digital natives' may have little to no memory of life before the internet. Their lives have been defined by social media and widespread connectivity and accessibility. In a 2018 Pew Research Center study, ${ }^{8} 95 \%$ of $13-17$-year-olds reported having a smartphone, with ownership near universal across different races and social backgrounds. $45 \%$ reported being online 'almost constantly' and $44 \%$ reported going online several times a day.

Although there are limited data on the technology-related healthcare preferences of AYA, some studies demonstrate that young adults want to access their health data online $e^{9-11}$ and as they get older, a greater proportion of them is anticipated to use patient portals. ${ }^{12,13}$ Safety experts advocate engaging patients with transparent notes, ${ }^{14,15}$ but attaining the potential benefits that come with reading notes requires raising awareness and actual note use by patients, ideally starting when they are young. These individuals may also be ideal advocates for shaping how health technology should continue to evolve in a patient-centred way. In this study, we surveyed patients at two large northeast USA academic medical centres, one focused on adult care and one specialised in paediatric care, and compared the responses of AYA to those older than 25 years old ('adults') to determine:

$>$ note-reading practices and perceptions regarding the importance of reading notes to engage in care

$>$ patient-provider relational effects of reading notes

$>$ safety and quality-related outcomes including, first, behaviours linked to tests, referrals, and medication adherence, and, second, experiences with note accuracy and reporting errors. 


\section{Method}

\section{Participants}

Portal account holders at the adult hospital and the paediatric hospital were eligible to participate if they had logged onto their portal at least once and had at least one available open note within the year prior to the study period (June 2016 to September 2016). At the paediatric hospital, individuals 13 years and older can register for their own portal account, and accounts are differentiated between parent/guardian accounts versus 'self' patient accounts. We invited a random half of eligible patients at the adult hospital to complete the survey through the patient portal, in compliance with portal-based survey restrictions at that hospital. At the paediatric hospital, we sent invitations to all eligible accounts, but we included only patient self-account holders in this analysis. Ten iPads were raffled off at each hospital as incentive for completing the survey.

\section{Survey development}

An interdisciplinary team including patient and family advocates developed the survey, which then underwent external validation by additional clinicians, patients and a survey scientist with expertise in patient engagement. Details about the survey's development are available elsewhere ${ }^{5}$ and the full survey is available upon request.

In the survey, we defined 'open notes' as 'the notes written by your healthcare provider(s) about your healthcare visits that you can read online' and distinguished notes from the patient portal itself by including a screenshot of the 'Notes' tab. We asked respondents how many visit notes they had read in the past 12 months. We queried those who reported 'none' about why they did not read any notes, with a list of potential barriers derived from previously published surveys. ${ }^{2}$

\section{Importance of note-reading for patient engagement} We evaluated the perceived importance of reading notes for four patient engagement behaviours in items using a 0-10 Likert scale, where 9-10 was defined as 'extremely important' as in prior published studies. ${ }^{16}$

\section{Relational items}

Questions about the influence of reading notes on the patientprovider relationship focused on trust, teamwork, goal alignment and activation, and used 'less/no change/more' response options (ie 'I trust my provider less/No change/I trust my provider more').

\section{Patient safety and quality}

Safety behaviour items focused on understanding and completing tests and referrals, which are common challenges in the ambulatory setting. ${ }^{17,18}$ First we asked participants if they had tests, referrals, or medication prescriptions in the prior year. Then we asked if they had a note addressing these issues. The survey was designed with skip logic so that only participants who had notes discussing these issues received questions about the impact of reading notes on each respective topic; for example, 'By reading your notes, were you more likely to check your tests results?' These questions had categorical responses (not at all/a little bit/quite a bit/very much). This survey strategy allowed us to evaluate actual experiences of reading notes rather than theoretical responses.

We asked all respondents about reporting attitudes such as 'Reporting mistakes improves patient safety,' using categorical answers (disagree/somewhat disagree/somewhat agree/agree/ don't know). We asked respondents who had read at least one note if they ever found an error in their notes and if so, how serious the error was (not at all/somewhat/very serious). We defined a 'serious mistake' as 'a problem that could have immediate effects on your care'. We combined participant responses of 'somewhat serious' and 'very serious' together, and refer to these as 'serious' errors from this point forward. We asked those who reported a serious error whether they contacted their provider's office about the error. If they did not, we asked why they would hesitate to speak up about the mistake, offering multiple options from a list of barriers derived from the literature. $^{19,20}$

Because we anticipated that a substantial proportion of respondents would not report having found an error in their notes in the past, ${ }^{21}$ we also asked how comfortable they would feel reporting a serious mistake if they found one in the future. Respondents who reported feeling less comfortable speaking up ( $\leq 7$ on a $0-10$ Likert scale) were asked why they would hesitate to speak up and could choose multiple options from a list of barriers derived from the literature, as previously described.

\section{Analysis}

We used descriptive statistics for sociodemographic characteristics and to report proportions of respondents in each group who identified a documentation error, and who contacted their provider about it. We used chi-squared analysis to examine the relationship between speaking up about the perceived error and record usage, defined as number of notes read by all patients in the study population. We calculated odds ratios (OR) and $95 \%$ confidence intervals (CIs) for the difference between AYA and adult responses related to 15 outcomes in three key domains of interest: importance of notes to engage in care (five items); relational effects of note-reading (four items); and safety behaviours related to note-reading (six items). Because we anticipated that the overall number of AYA identifying a serious mistake would be small (based on the literature and the population size), we did not apply statistical testing to compare results to adults and instead report experiences with note accuracy and reporting errors descriptively. ${ }^{21,22}$

\section{Ethics}

This study was approved by each hospital's institutional review board: BIDMC 2016P000045 and BCH IRB-P0002184.

\section{Results}

\section{General characteristics}

In total, 6,913 of 24,722 ( $28 \%$ ) sampled account holders at the adult hospital and 3,672 of $21,579(17 \%)$ account holders at the paediatric hospital completed the survey. Among the paediatric hospital respondents, 3,339 were parents or guardians of 


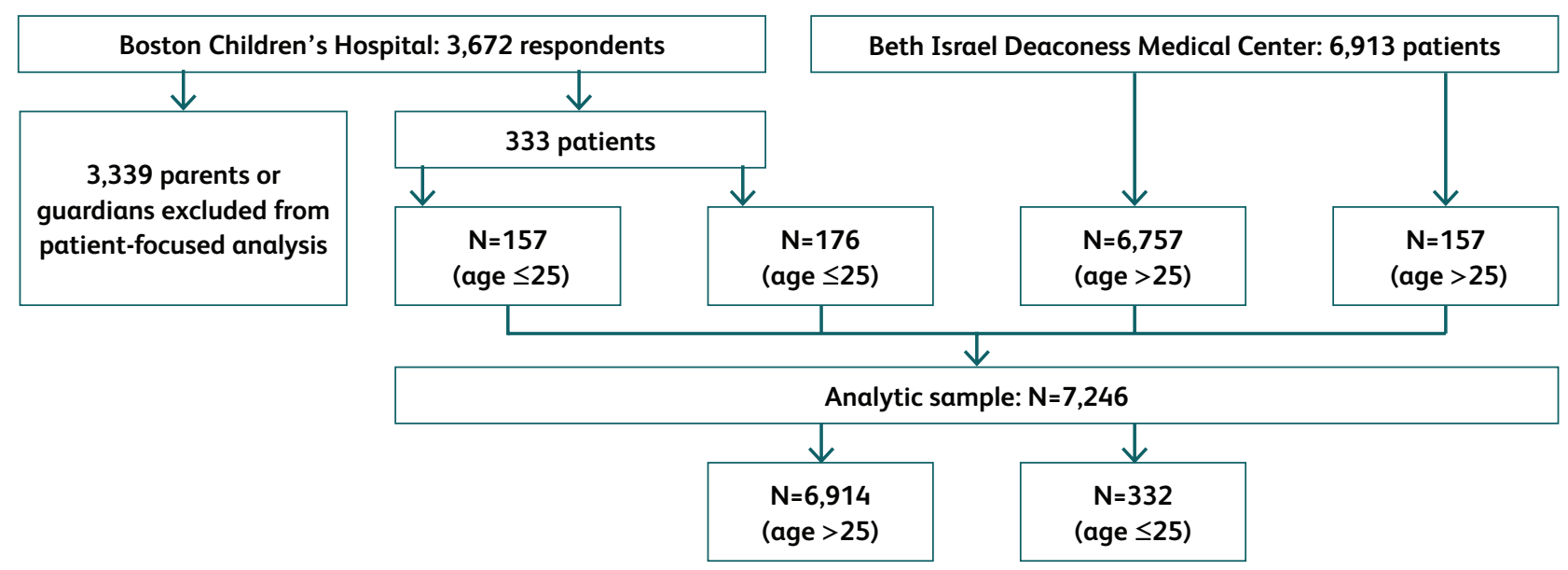

Fig 1. Study population flowchart.

paediatric patients and 333 were self-account holders. Parents/ guardians were excluded from our analysis. In total, 7,246 patients were included in this study (Fig 1); 332 participants from both sites were AYA (13-25 years old) and 6,914 respondents were adults $(>25$ years old).

Table 1 shows demographic characteristics of the participants. The mean age of AYA was $22+/-2.7$, while the mean age of the adult group was $56+1-14$. The majority of respondents were white (78\% AYA, $89 \%$ adults) and non-Hispanic (92\%; 97\%). While most respondents rated their health as very good or excellent, $13 \%$ of AYA and $12 \%$ of adults reported their health as fair or poor. Roughly one-third of both groups reported $\geq 6$ healthcare visits during the prior year (32\% AYA, $40 \%$ adults).

\section{Note reading behaviour and importance of notes to engage in care}

The majority of participants in both groups read notes, with $88 \%$ of AYA and $95 \%$ of adults reporting they read at least one note the past year (Table 1). Among those who did not read at least one note, the most common reasons were similar between groups: '[I] forgot my notes are available' (45\% AYA; $43 \%$ adults), 'difficulties using portal' (10\%; $8 \%$ ), and 'too busy' (10\%; $8 \%$ ). AYA were as likely as adults to agree that reading notes was 'extremely important' to feel informed (61\% AYA, 63\% adults), understand their provider's thinking $(54 \% ; 60 \%)$, remember the plan of care (42\%; $44 \%)$, and make decisions about their care ( $40 \%$; 44\%). All OR and $95 \%$ CIs comparing note importance between groups showed no significant difference, demonstrating similar benefits reported between the 2 groups (Table 2).

\section{Relational effects}

About half of AYA felt that reading notes improved their trust, teamwork, activation and goal alignment with their provider (Table 3). Roughly half of patients across all ages reported no change in the relationship with their provider after reading notes. Very few participants in either group reported negative relational effects from reading notes. Comparison of respondent groups showed no significant differences for each relational item (Table 3 ).

\section{Patient safety}

\section{Safety knowledge and behaviours}

AYA and adults reported that reading notes helped them with safety and quality-related behaviours involving tests, referrals and medication adherence (Table 4). A majority in each group reported that reading notes helped them quite a bit or very much to understand why their providers ordered tests (70\% AYA; $74 \%$ adults), check test results $(76 \% ; 79 \%)$, understand test results $(74 \% ; 74 \%)$, and understand the rationale for their referrals $(77 \% ; 75 \%)$. Similar proportions of AYA as adults reported that reading notes helped them quite a bit or very much to remember to: get their tests done (39\% AYA; $45 \%$ adults), go to referral appointments (51\%; 54\%), and take medications as prescribed $(36 \% ; 36 \%)$. OR and $95 \%$ CI comparisons between groups demonstrated no significant differences in patient-reported benefits in any of the items (Table 4).

\section{Attitudes about patient safety and error reporting}

Nearly all respondents felt it was important to identify and speak up about errors. Safety attitudes between AYA and adults were strikingly similar, including agreeing with the following statements: reporting mistakes improves patient safety (99\% AYA; $98 \%$ adults); patients can play a role in preventing mistakes in their care by paying attention to what is in the medical record (97\%; $98 \%$ ); and providers want to know about mistakes patients find in their medical records $(88 \% ; 89 \%)$.

Twenty-five to $30 \%$ of all participants reported a possible mistake in their notes; $10 \%$ of AYA and $18 \%$ of adults reported finding a mistake in their notes; an additional $15 \%$ of AYA and $12 \%$ of adults were not sure. Among those who identified an error, about 40\% (39\% AYA; 44\% adults) reported that the most important mistake they found was serious. Overall, about half of all respondents contacted their doctor about the perceived serious error: AYA were somewhat less likely to speak up about the mistake than adults ( $43 \%$ versus $58 \%$ ). The two most common reasons cited by AYA were, 'Nothing would be done about it' (100\%) and 'I don't want to be thought of as "difficult" or a "troublemaker"' $(100 \%)$. The most common reason adults selected was, 'I didn't 
Table 1. Demographic characteristics of participants

\begin{tabular}{|c|c|c|c|}
\hline Characteristic & $\begin{array}{l}\text { Overall } \\
n=7,246\end{array}$ & $\begin{array}{l}\text { Adolescents and } \\
\text { young adults } n=332\end{array}$ & $\begin{array}{l}\text { Adults } \\
\mathrm{n}=6,914\end{array}$ \\
\hline Mean age $(+/-S D)$ & $54.6(15.6)$ & $21.5(2.7)$ & $56.2(14.1)$ \\
\hline \multicolumn{4}{|l|}{ Sex } \\
\hline Female & $4,343(62.8 \%)$ & $128(82.1 \%)$ & $4,215(62.4 \%)$ \\
\hline Male & $2,570(37.2 \%)$ & $28(18.0 \%)$ & $2,542(37.6 \%)$ \\
\hline \multicolumn{4}{|l|}{ Race } \\
\hline Asian & $293(4.8 \%)$ & $20(15.3 \%)$ & $273(4.6 \%)$ \\
\hline Black or African American & $229(3.7 \%)$ & $11(8.4 \%)$ & $218(3.6 \%)$ \\
\hline White & $5,448(88.9 \%)$ & $97(74.1 \%)$ & $5,351(89.1 \%)$ \\
\hline Other $^{a}$ & $165(2.7 \%)$ & $3(2.3 \%)$ & $162(2.7 \%)$ \\
\hline \multicolumn{4}{|l|}{ Ethnicity } \\
\hline Hispanic or Latino & $229(3.7 \%)$ & $15(7.9 \%)$ & $214(3.5 \%)$ \\
\hline \multicolumn{4}{|l|}{ Self-reported health } \\
\hline Excellent or very good & $3,521(55.7 \%)$ & $106(55.2 \%)$ & $3,415(55.7 \%)$ \\
\hline Good & $2,026(32.0 \%)$ & $62(32.3 \%)$ & $1,964(32.0 \%)$ \\
\hline Fair or poor & $781(12.3 \%)$ & $24(12.5 \%)$ & $757(12.3 \%)$ \\
\hline \multicolumn{4}{|l|}{ Highest level of education ${ }^{b}$} \\
\hline$\leq$ High school graduate & $1,018(16.2 \%)$ & $38(19.9 \%)$ & $980(16.0 \%)$ \\
\hline 2- or 4-year college graduate & $2,207(35.0 \%)$ & $103(53.9 \%)$ & $2,104(34.4 \%)$ \\
\hline Graduate school, Masters, or Doctoral degree & $3,080(48.9 \%)$ & $50(26.2 \%)$ & $3,030(49.6 \%)$ \\
\hline \multicolumn{4}{|l|}{$\begin{array}{l}\text { Number of visits to any healthcare facility } \\
\text { over last } 12 \text { months }\end{array}$} \\
\hline 0 & $40(0.6 \%)$ & $4(2.1 \%)$ & $36(0.6 \%)$ \\
\hline $1-5$ & $3,729(58.9 \%)$ & $126(65.6 \%)$ & $3,603(58.7 \%)$ \\
\hline 6 or more & $2,559(40.4 \%)$ & $62(32.3 \%)$ & $2,497(40.7 \%)$ \\
\hline \multicolumn{4}{|l|}{$\begin{array}{l}\text { About how many visit notes have you read } \\
\text { in the past } 12 \text { months? }\end{array}$} \\
\hline None & $227(3.2 \%)$ & $20(9.1 \%)$ & $207(3.0 \%)$ \\
\hline 1 only & $501(7.1 \%)$ & $29(13.1 \%)$ & $472(7.0 \%)$ \\
\hline 2 or more & $6,135(87.3 \%)$ & $165(74.7 \%)$ & $5,970(87.8 \%)$ \\
\hline Don't know / Don't remember & $162(2.3 \%)$ & $7(3.2 \%)$ & $155(2.3 \%)$ \\
\hline
\end{tabular}

a' Other' races include: American Indian, Pacific Native, Native Hawaiian, Pacific Islander, and multiple races.

${ }^{\text {b} E d u c a t i o n a l ~ s t a t u s ~ r e p o r t i n g ~ m a y ~ b e ~ i n t r i n s i c a l l y ~ l i m i t e d ~ b y ~ a g e ~ o f ~ a d o l e s c e n t s ~ a n d ~ y o u n g ~ a d u l t s . ~}$

know I could report a mistake' (59\%). Although respondents who read more notes trended toward more likely contacting their provider's office about the mistake, the difference was not significant $(p=0.19)$

AYA were also less likely to report that they would feel very comfortable reporting a serious mistake if they found one in the future compared to adults ( $65 \%$ versus $79 \%$ ). The most common barrier to notifying the provider about a hypothetical (future) serious mistake was not knowing they could report a mistake $(72 \%$ AYA; $52 \%$ adults). For adults, the three next most common barriers were not wanting to be thought of as 'difficult' or a 'troublemaker' $(34 \%)$, feeling unsure that the mistake is important (24\%), and concern that their provider is too busy ( $23 \%$ ). In contrast, AYA were more concerned about not wanting to be thought of as 'difficult' or a 'troublemaker' $(47 \%)$, worrying that they will annoy their provider $(28 \%)$, and not knowing whom to call or talk to $(25 \%)$. 
Table 2. Participant perceptions about the importance of notes to engage in care

Overall

$n=7,246$

How important is reading your notes in feeling informed about your care?

$0-5$

$6-8$

$9-10$

\section{Adolescents and}

young adults $n=332$

Adults

$n=6,914$

Odds ratio ${ }^{a}$

(95\% CI)

$0.9(0.7,1.3)$

$623(9.6 \%)$

$1,766(27.2 \%)$

$4,093(63.1 \%)$
$17(9.3 \%)$

$54(29.5 \%)$

$112(61.2 \%)$
$606(9.6 \%)$

$1,712(27.2 \%)$

$3,981(63.2 \%)$

How important is reading your notes in understanding how your provider(s) are thinking about your medical conditions?

$0-5$

6-8

9-10

How important is reading your notes in remembering the plan for your care?

$0-5$

6-8

9-10

How important is reading your notes in helping you make decisions about your care?

$0-5$

6-8

9-10
$715(11.0 \%)$

$1,874(28.9 \%)$

$3,892(60.1 \%)$

$695(11.0 \%)$

$1,809(28.7 \%)$

$3,794(60.2 \%)$

$\begin{array}{lll}1,768(27.3 \%) & 51(27.9 \%) & 1,717(27.3 \%) \\ 1,842(28.4 \%) & 55(30.1 \%) & 1,787(28.4 \%) \\ 2,870(44.3 \%) & 77(42.1 \%) & 2,793(44.4 \%)\end{array}$

$0.8(0.6,1.1)$

$0.9(0.7,1.2)$

'The odds ratio was calculated based on the outcome of interest - notes are 'extremely important' - defined as odds of '9-10' versus other combined categories in AYA compared with adults.

In both groups, participants reported that if they found an error in their notes, they would prefer to call the provider who wrote the note ( $56 \%$ AYA; $55 \%$ adults) or submit a request for a correction online $(54 \% ; 54 \%)$. A significant portion also preferred to talk to their provider about it at the next visit $(37 \% ; 31 \%)$. AYA were somewhat more likely to want to speak to someone other than the provider ( $24 \%$ versus $9 \%$ ) or call the nurse in the office $(24 \%$ versus $18 \%$ ).

\section{Discussion}

Adolescents and young adults are a unique population that grew up immersed in an 'always connected' world. As regulations expand their access to health data, a better understanding of how young adults interact with open notes can help safety leaders learn to partner with them in their care. Although patient portals may be more 'clunky' than the typical technologies that AYA engage with, our study of over 7,000 patients showed that the majority of AYA and adults read notes. In addition, AYA were as likely as adults to report that reading notes is extremely important for feeling engaged in their care, that it improved their relationship with their provider, and helped them remember key safety behaviours related to tests, referrals, and medication adherence, with no significant differences between groups in 15/15 outcomes within these three study domains of interest. These findings suggest that AYA are just as likely to benefit from note-reading as adults and that they should be encouraged by their providers to access notes and read them as a patient safety and engagement strategy.

About half of AYA respondents reported that reading notes improved their relationship with their provider and increased trust, consistent with a prior study on the effects of reading notes (with the majority of the remainder reporting no change). ${ }^{7}$ This finding is especially important in a population that regularly uses the internet to search for health topics and looks to providers to validate information. ${ }^{11,23,24}$ In one survey, ${ }^{25}$ only $25 \%$ were satisfied with the information they found online. Sharing notes can help AYA find reliable information about their health conditions provided by their own clinicians.

Prior studies have shown that young adults are less likely than adults to review appointments and laboratory test ${ }^{26}$ and that young adults struggle with understanding their medications. ${ }^{11}$ Our findings suggest that reading notes may help overcome these barriers. Strikingly, $75 \%$ of AYA and adults reported that reading their notes helped them understand why their provider ordered tests and referrals, and also helped them remember to look up and understand the results of their tests. Over one-third AYA reported that note-reading helped them with medication adherence, a 
Table 3. Effect of reading notes on patient-provider relationship

\section{Trust in provider}

I trust my provider less

No change

I trust my provider more

\section{Teamwork between patient} and provider

We are working together less as a team

No change

We are working together more as a team

Alignment between provider's goals and patient's goals for health care

My provider's goals match my own goals less

No change

My provider's goals match my own goals more

\section{Activation in health care}

I am less active in my health care

No change

I am more active in my health care

\section{Overall \\ $\mathrm{n}=7,246$}

Adolescents and
young adults
$n=332$

Adults $\mathrm{n}=6,914$

$n=332$
$183(2.8 \%)$

$3,582(54.2 \%)$

$2,846(43.1 \%)$
$10(5.4 \%)$

$83(44.4 \%)$

$94(50.3 \%)$
Odds ratio ${ }^{a}$ (95\% CI)

$1.3(0.9,1.7)$

$173(2.7 \%)$

$3,499(54.5 \%)$

$2,752(42.8 \%)$

$0.8(0.6,1.1)$

$\begin{array}{ll}7(3.7 \%) & 128(2.0 \%) \\ 104(55.6 \%) & 3,350(52.2 \%) \\ 76(40.6 \%) & 2,943(45.8 \%)\end{array}$

$7(3.7 \%)$

$135(2.0 \%)$

$3,454(52.3 \%)$

$3,019(45.7 \%)$

$76(40.6 \%)$

$0.9(0.7,1.2)$

$12(6.4 \%)$

$176(2.7 \%)$

$99(52.9 \%)$

$3,662(57.0 \%)$

$76(40.6 \%)$

$2,585(40.3 \%)$
$3,761(56.9 \%)$

$2,661(40.3 \%)$

$38(0.6 \%)$

3,563 (53.9\%)

$3,012(45.6 \%)$
$1(0.5 \%)$

$99(52.9 \%)$

$87(46.5 \%)$
$1.0(0.8,1.4)$

$37(0.6 \%)$

$3,464(53.9 \%)$

$2,925(45.5 \%)$

'The odds ratio was calculated based on the outcome of interest - the odds of 'more' trust/teamwork/alignment/activation versus other combined categories in AYA compared with adults

notable finding during this pivotal time of shifting healthcare responsibility from parents of paediatric patients to young adults.

The overwhelming majority of respondents in both groups agreed that reporting mistakes improves safety outcomes, but AYA were somewhat less likely to report a serious mistake they found in their notes, or to feel very comfortable speaking up about a hypothetical serious mistake in their notes in the future. Both age groups reported not knowing that they could report a mistake as a key barrier, underscoring the importance of encouraging patients to speak up about potential errors they find in their notes and providing clear instructions for how to do so. AYA were more likely to worry about being a 'troublemaker' or annoying their provider which may reflect concerns related to 'newer' relationships with providers, or generational differences regarding conflict avoidance. AYA may feel more uncertain about communicating with their providers ${ }^{26}$ and may benefit from additional encouragement to speak up. AYA may also need guidance and education about how to identify errors and what is important to report.

There are several limitations to this study. The response rate was low, although not dissimilar from published online surveys. ${ }^{27,28}$ A relatively small proportion of participants were 25 years of age or younger. Although the majority of respondents rated their health as very good or excellent, about one-third of both age groups reported multiple visits in the prior year, suggesting our study captured a population with chronic illnesses who see their providers regularly. These AYA may be more likely to hold portal accounts, may be more likely to read notes, and may not have the same attitudes and preferences as the average healthy young patient. Our results also reflect findings from prior studies that the majority of AYA portal users are white and those who have had multiple provider visits. ${ }^{12,29}$ Further research into how other populations interact with the portal and use notes is needed.

\section{Conclusion}

Today's adolescents and young adults have grown up immersed in technology, but their interest in and benefit from reading their care notes online is not well understood. In our study, the majority of AYA read notes and rated them as extremely important for several engagement and safety behaviours. Although a similar proportion of AYA reported definite or possible errors in their notes as adults, fewer spoke up about them, citing knowledge and cultural barriers like fear of conflict. Taken together, these findings support initiatives that encourage AYA to read notes and share concerns. Efforts that include note-reading may help transition patients from paediatric to adult care with greater autonomy, activation and safety partnership. 
Table 4. Effect of reading notes on safety knowledge and behaviours

\section{Overall \\ $n=7,246$}

Has reading your notes helped you understand why your provider(s) ordered tests?

Not at all

A little bit

Quite a bit or very much

Has reading your notes helped you remember to get tests done?

Not at all

A little bit

Quite a bit or very much

By reading your notes, were you more likely to check your test results?

Not at all

A little bit

Quite a bit or very much

Has reading your notes helped you understand the test results?

Not at all

A little bit

Quite a bit or very much

Has reading your notes helped you understand the reason for the referral?

Not at all

A little bit

Quite a bit or very much

Has reading your notes helped you remember to go to your appointment with the specialist or other healthcare provider?

Not at all

A little bit

Quite a bit or very much

Has reading your notes helped you take your medications better as prescribed?

Not at all

$1,546(41.9 \%)$
$830(22.5 \%)$
$1,313(35.6 \%)$

$1,742(32.9 \%)$

$1,179(22.3 \%)$

$2,374(44.8 \%)$

$422(8.0 \%)$

$679(12.8 \%)$

$4,192(79.2 \%)$

Adolescents and young adults $\mathrm{n}=332$

Adults

$n=6,914$

Odds ratio ${ }^{a}$ (95\% CI)

$0.8(0.6,1.3)$

$347(6.7 \%)$

$1,016(19.7 \%)$

$3,802(73.6 \%)$

$0.9(0.6,1.2)$

1,699 (32.9\%)

$1,143(22.1 \%)$

$2,324(45.0 \%)$

$0.9(0.6,1.3)$

$7(5.4 \%)$

$415(8.0 \%)$

$655(12.7 \%)$

4,094 $(79.3 \%)$

$1.1(0.7,1.6)$

$245(4.6 \%)$

$4(3.1 \%)$

$241(4.7 \%)$

$1,152(21.8 \%)$

$29(22.5 \%)$

$1,123(21.8 \%)$

$3,895(73.6 \%))$

$96(74.4 \%)$

$3,799(73.6 \%)$

$1.2(0.6,2.5)$

$\begin{array}{ll}202(8.4 \%) & 0(0.0 \%) \\ 387(16.2 \%) & 10(23.3 \%) \\ 1,805(75.4 \%) & 33(76.7 \%)\end{array}$

$202(8.6 \%)$

$377(16.0 \%)$

$1,772(75.4 \%)$

$1.1(0.6,2.0)$

$\begin{array}{ll}660(27.6 \%) & 7(16.3 \%) \\ 447(18.7) & 14(32.6 \%) \\ 1,285(53.7 \%) & 22(51.2 \%)\end{array}$

$653(27.8 \%)$

$433(18.4 \%)$

$1,263(53.8 \%)$

$1.1(0.8,1.6)$

$1,313(35.6 \%)$

$$
\begin{aligned}
& 43(38.1 \%) \\
& 29(25.7 \%) \\
& 41(36.3 \%)
\end{aligned}
$$$$
1,503(42.0 \%)
$$$$
801(22.4 \%)
$$

$1,272(35.6 \%)$

a The odds ratio was calculated as the outcome of interest - the odds of notes helping patients with safety knowledge and behaviours 'quite a bit' or 'very much' versus other combined categories in AYA compared with adults.

The total $\mathrm{n}$ for any given item varied based on the number of patients who had tests, referrals, and medication prescriptions as well as notes describing those issues. 


\section{References}

1 Health and Human Services Department. 21st century cures act: Interoperability, information blocking, and the ONC Health IT certification program. Federal Register, 2020.

2 Delbanco T, Walker J, Bell SK et al. Inviting patients to read their doctors' notes: a quasi-experimental study and a look ahead. Ann Intern Med 2012;157:461-70.

3 Walker J, Darer JD, Elmore JG, Delbanco T. The road toward fully transparent medical records. N Engl J Med 2014;370:6-8.

4 Nazi KM, Turvey CL, Klein DM, Hogan TP, Woods SS. VA OpenNotes: exploring the experiences of early patient adopters with access to clinical notes. J Am Med Inform Assoc 2015;22:380-9.

5 Bell SK, Folcarelli P, Fossa A et al. Tackling ambulatory safety risks through patient engagement: What 10,000 patients and families say about safety-related knowledge, behaviors, and attitudes after reading visit notes. J Patient Saf 2018, in press (doi: 10.1097/ PTS.0000000000000494).

6 Huang JS, Yueh R, Ma S et al. Adolescents' and young adults' satisfaction with and understanding of medical notes from a pediatric gastroenterology practice: A cross-sectional cohort study. J Pediatr 2019;215:264-6.

7 Dohil I, Cruz R, Sweet H, Huang JS. Sharing notes with adolescents and young adults admitted to an inpatient psychiatry unit. J Am Acad Child Adolesc Psychiatry 2021;60:317-20.

8 Anderson M, Jiang J. Teens, social media and technology 2018. Pew Research Center, 2018.

9 Safavi K, Kalis B. Accenture 2019 digital health consumer survey. Accenture, 2019.

10 Bergman DA, Brown NL, Wilson S. Teen use of a patient portal: a qualitative study of parent and teen attitudes. Perspect Health Inf Manag 2008;5:13.

11 Applebaum MA, Lawson EF, von Scheven E. Perception of transition readiness and preferences for use of technology in transition programs: teens' ideas for the future. Int J Adolesc Med Health 2013;25:119-25.

12 Steitz B, Cronin RM, Davis SE, Yan E, Jackson GP. Long-term Patterns of Patient Portal Use for Pediatric Patients at an Academic Medical Center. Appl Clin Inform 2017:8:779-93.

13 Szilagyi PG, Valderrama R, Vangala S et al. Pediatric patient portal use in one health system. J Am Med Inform Assoc 2020;27:444-8.

14 Balogh EP, Miller BT, Ball JR (eds). Improving diagnosis in health care. National Academies Press, 2015.

15 National Steering Committee for Patient Safety. Safer together: A national action plan to advance patient safety. Institute for Healthcare Improvement, 2020.

16 Gerard M, Chimowitz H, Fossa A et al. The Importance of Visit Notes on Patient Portals for Engaging Less Educated or Nonwhite Patients: Survey Study. J Med Internet Res 2018;20:e191.
17 Partnership for Health IT Patient Safety. Health IT safe practices for closing the loop. ECRI Institute, 2017. www.ecri.org/Resources/ HIT/Closing_Loop/Closing_the_Loop_Toolkit.pdf

18 Gandhi TK, Kachalia A, Thomas EJ et al. Missed and delayed diagnoses in the ambulatory setting: a study of closed malpractice claims. Ann Intern Med 2006;145:488-96.

19 Bell SK, Roche SD, Mueller A et al. Speaking up about care concerns in the ICU: patient and family experiences, attitudes and perceived barriers. BMJ Qual Saf 2018;27:928-36.

20 Lam BD, Bourgeois F, Dong ZJ, Bell SK. Speaking up about patientperceived serious visit note errors: Patient and family experiences and recommendations. J Am Med Inform Assoc 2021;28:685-94.

21 Bell SK, Delbanco T, Elmore JG et al. Frequency and types of patient-reported errors in electronic health record ambulatory care notes. JAMA Netw Open 2020;3:e205867.

22 Bourgeois FC, Fossa A, Gerard M et al. A patient and family reporting system for perceived ambulatory note mistakes: experience at 3 U.S. healthcare centers. J Am Med Inform Assoc 2019:26:1566-73.

23 Radovic A, McCarty CA, Katzman K, Richardson LP. Adolescents' perspectives on using technology for health: qualitative study. JMIR Pediatr Parent 2018;1:e2.

24 Skinner H, Biscope S, Poland B, Goldberg E. How adolescents use technology for health information: implications for health professionals from focus group studies. J Med Internet Res 2003;5:e32.

25 Wartella E, Rideout V, Montague H, Beaudoin-Ryan L, Lauricella A. Teens, health and technology: a national survey. Media Commun 2016;4:13-23.

26 Thompson LA, Martinko T, Budd P, Mercado R, Schentrup AM. Meaningful Use of a Confidential Adolescent Patient Portal. J Adolesc Health 2016;58:134-40.

27 Forcino RC, Barr PJ, O'Malley AJ et al. Using CollaboRATE, a brief patient-reported measure of shared decision making: Results from three clinical settings in the United States. Health Expect 2018;21:82-9.

28 Medicaid. Methodology report: 2014-2015 nationwide CAHPS survey of adults enrolled in Medicaid between October and December, 2013. Medicaid, 2015. www.medicaid.gov/medicaid/ quality-of-care/downloads/performance-measurement/methodology-report.pdf [Accessed 5 September 2020].

29 Ketterer T, West DW, Sanders VP et al. Correlates of patient portal enrollment and activation in primary care pediatrics. Acad Pediatr 2013;13:264-71.

Address for correspondence: Barbara D Lam, 330 Brookline Avenue, Boston, MA, 02215, USA.

Email: sblam@bidmc.harvard.edu 